\title{
Operator product expansion and conservation laws in non-relativistic conformal field theories
}

\author{
Siavash Golkar and Dam T. Son \\ Kadanoff Center for Theoretical Physics and \\ Enrico Fermi Institute, University of Chicago, \\ 5640 South Ellis Ave., Chicago, IL, 60637 U.S.A. \\ E-mail: golkar@uchicago.edu, dtson@uchicago.edu
}

\begin{abstract}
We explore the consequences of conformal symmetry for the operator product expansions in nonrelativistic field theories. Similar to the relativistic case, the OPE coefficients of descendants are related to that of the primary. However, unlike relativistic CFTs the 3-point function of primaries is not completely specified by conformal symmetry. Here, we show that the 3 -point function between operators with nonzero particle number, where (at least) one operator has the lowest dimension allowed by unitarity, is determined up to a numerical coefficient. We also look at the structure of the family tree of primaries with zero particle number and discuss the presence of conservation laws in this sector.
\end{abstract}

KEYwords: Conformal and W Symmetry, Space-Time Symmetries

ARXiv EPRINT: 1408.3629 


\section{Contents}

1 Introduction 1

2 Nonrelativistic conformal symmetry 2

2.1 The Schrödinger algebra 2

2.2 Correlators 3

3 The operator product expansion 3

4 Restrictions on multipoint correlators 5

$\begin{array}{lll}5 & \text { Zero particle number sector and conservation laws } & 7\end{array}$

$\begin{array}{llr}6 & \text { Conclusion } & 9\end{array}$

\section{Introduction}

Experimental studies of fermions at unitarity have stimulated theoretical developments of nonrelativistic conformal field theories. The conformal extension of the Galilean algebra was found a long time ago $[1,2]$ and was later analyzed in the context of string theory. Mehen, Stewart, and Wise explored the consequences of the conformal invariance for the scattering amplitudes involving unitarity fermions [3], and subsequently other applications have been considered in the literature [4-6].

The understanding of the operator structure of nonrelativistic conformal field theory is still not complete. Only recently has the operator product expansion begun to be explored. The motivation was to probe unitarity fermions at short distances. There have also been attempts to construct holographic duals of the unitarity fermions [7-9]. A very interesting nontrivial check is the computation of the three-point function from hologrpahy, which yields the same result as the calculation in the field theory of unitarity fermions.

In this paper we explore the consequences of conformal invariance on the structure of the OPEs. One property of nonrelativistic OPEs is that the OPE coefficients involves, in general, nontrivial functions of the ratio $\mathbf{x}^{2} / t$, where $\mathbf{x}$ and $t$ are the separation between the two points:

$$
O_{1}(x) O_{2}(0)=\sum \frac{1}{|\mathbf{x}|^{\Delta_{1}+\Delta_{2}-\Delta_{n}}} c_{n}\left(\frac{x^{2}}{t}\right) O_{n}(0) .
$$

Here and later, $x \equiv(t, \mathbf{x})$, but $x^{2} \equiv|\mathbf{x}|^{2}$.

In general conformal symmetry is not powerful enough to restrict the form of the functions $c_{n}$. In this work, however, we show that when one of the operators participating in the OPE is an elementary field, that is it carries scaling dimension equal to $d / 2$, the 


\begin{tabular}{|c|c|c|c|c|c|}
\hline $\mathrm{A}$ & $P_{j}$ & $K_{j}$ & $D$ & $C$ & $H$ \\
\hline$P_{i}$ & 0 & $-i \delta_{i j} N$ & $-i P_{i}$ & $-i K_{i}$ & 0 \\
\hline$K_{i}$ & $i \delta_{i j} N$ & 0 & $i K_{i}$ & 0 & $i P_{i}$ \\
\hline$D$ & $i P_{j}$ & $-i K_{j}$ & 0 & $-2 i C$ & $2 i H$ \\
\hline$C$ & $i K_{j}$ & 0 & $2 i C$ & 0 & $i D$ \\
\hline$H$ & 0 & $-i P_{j}$ & $-2 i H$ & $-i D$ & 0 \\
\hline
\end{tabular}

Table 1. Values of $[A, B]$.

OPE coefficents are, in general, determined up to a numerical coefficient. The exception is when one of the other operator has particle number zero, in which case not all the OPE coefficients are fixed by symmetry.

We also look at the structure of the family generated by a primary with particle number zero. This case is particular because the a subset of the ladder operators in the algebra commute when the particle number is zero. We discuss the consequences of this degeneracy and its implications for the existence of conservation laws.

\section{Nonrelativistic conformal symmetry}

To make the paper self-contained, we recall some basic fact about nonrelativistic conformal field theories [6].

\subsection{The Schrödinger algebra}

The Schrödinger algebra in $d$ dimensions, $\mathfrak{s c h}_{d}$ is formed from the operators $N, D, M_{i j}$, $K_{i}, P_{i}, C$ and $H$, respectively the number (mass), scaling, rotation, Galilean boost, spatial translation, special Schrödinger transformation and time translation operators. The operator $N$ is central and all operators transform with the appropriate tensor structure under rotations $M_{i j}$. The rest of the algebra is given in table 1 .

We look at representations made of local operators such that:

$$
\mathcal{O}(x)=e^{i H t-i \mathbf{P} \cdot \mathbf{x}} \mathcal{O}(0) e^{-i H t+i \mathbf{P} \cdot \mathbf{x}} .
$$

Since $N$ is central, it is also convenient to look at operators that have specific particle number $N_{\mathcal{O}}$ :

$$
[N, \mathcal{O}(0)]=N_{\mathcal{O}} \mathcal{O}(0) .
$$

If we look at operators $C, H$ and $D$, they span a subalgebra of $\mathfrak{s c h r}_{d}$ isomorphic to $\mathfrak{s u}(1,1)$ corresponding to the lowering, raising and diagonal operators repectively. An operator $\mathcal{O}$ is said to have scaling dimension $\Delta_{\mathcal{O}}$ if:

$$
[D, \mathcal{O}(0)]=i \Delta_{\mathcal{O}} \mathcal{O}(0)
$$

The operators $P_{i}$ and $K_{i}$ also act as raising and lowering for the eigenvalue of $D$, albeit with increments of 1 (compared to increments of 2 in the case of $H$ and $C$ ). We can therefore, 
classify the representations of $\mathfrak{s c h} \mathfrak{r}_{d}$ with their number and the lowest scaling dimension. (These form standard cyclic representations.) We will call the operator of lowest weight a primary operator:

$$
[C, \mathcal{O}]=0, \quad\left[K_{i}, \mathcal{O}\right]=0 .
$$

For primary operators, the action of $C$ and $K_{i}$ can be written as:

$$
\begin{aligned}
{\left[K_{i}, \mathcal{O}(x)\right] } & =\left(-i t \partial_{i}+N_{\mathcal{O}} x_{i}\right) \mathcal{O} \\
{[C, \mathcal{O}(x)] } & =-i\left(t^{2} \partial_{t}+x_{i} \partial_{i}+t \Delta_{\mathcal{O}}\right) \mathcal{O}+\frac{x^{2}}{2} N_{\mathcal{O}} \mathcal{O}
\end{aligned}
$$

\subsection{Correlators}

The Schrödinger algebra puts restrictions on the form of the correlators. Similar to relativistic CFTs the form of the 2-point function of primaries is determined upto an overall constant [4]. For scalar primaries we have (similar result holds for operators which transform like tensors with respect to rotations):

$$
\left\langle\mathcal{O}_{1}\left(t_{1}, \mathbf{x}_{1}\right) \mathcal{O}_{2}\left(t_{2}, \mathbf{x}_{2}\right)\right\rangle=c \delta_{\Delta_{1} \Delta_{2}} t_{12}^{-\Delta_{1}} \exp \left(\frac{i M}{2} \frac{\mathbf{x}_{12}^{2}}{t_{12}}\right)
$$

where $t_{12} \equiv t_{1}-t_{2}$ and $\mathbf{x}_{12} \equiv \mathbf{x}_{1}-\mathbf{x}_{2}$.

However, conformal symmetry does not completely fix the three-point function, which can depend on one arbitrary function (in the relativistic case, this occurs for four- and higher-point correlators). We have, for three- and four-point functions $[4,10]$ :

$$
\begin{aligned}
\left\langle\mathcal{O}_{1} \mathcal{O}_{2} \mathcal{O}_{3}\right\rangle= & \prod_{i<j} t_{i j}^{\frac{1}{2} \Delta-\left(\Delta_{i}+\Delta_{j}\right)} \exp \left(\frac{i M_{1}}{2} \frac{x_{13}^{2}}{t_{13}}+\frac{i M_{2}}{2} \frac{x_{23}^{2}}{t_{23}}\right) F_{3}\left(v_{123}\right) \\
\left\langle\mathcal{O}_{1} \mathcal{O}_{2} \mathcal{O}_{3} \mathcal{O}_{4}\right\rangle= & \prod_{i<j} t_{i j}^{\frac{1}{6} \Delta-\left(\Delta_{i}+\Delta_{j}\right) / 2} \exp \left(\frac{i M_{1}}{2} \frac{x_{14}^{2}}{t_{14}}+\frac{i M_{2}}{2} \frac{x_{24}^{2}}{t_{24}}+\frac{i M_{2}}{2} \frac{x_{34}^{2}}{t_{34}}\right) \times \\
& \times F_{4}\left(\frac{t_{12} t_{34}}{t_{13} t_{24}}, v_{124}, v_{134}, v_{234}\right)
\end{aligned}
$$

where $\Delta=\sum \Delta_{i}$ and:

$$
v_{i j k}=\frac{1}{2}\left(\frac{x_{j k}^{2}}{t_{j k}}-\frac{x_{i k}^{2}}{t_{i k}}+\frac{x_{i j}^{2}}{t_{i j}}\right) .
$$

$F_{3}$ and $F_{4}$ are functions of one and four variables respectively. The form of these functions is not not restricted by the symmetries.

\section{The operator product expansion}

Similar to relativistic CFTs [11-13], we expect the product of two operators at two different points to be expressible as a sum of local operators. Restricting to the OPEs of primary scalar operators (similar results hold for the OPE of tensor operators) we have:

$$
\mathcal{O}_{i}(t, x) \mathcal{O}_{j}(0)=\sum_{k, l} C_{i j}^{k l}(t, x) \mathcal{O}_{k, l},
$$


where $\mathcal{O}_{k, l}$ denotes the l'th descendant of the primary operator $\mathcal{O}_{k}$. The Schödinger algebra puts stringent restrictions on the form of the coefficients $C_{i j}^{k l}$, where in most cases, we can read off the coefficient of descendants from the coefficient of the primary $C_{i j}^{k 0}$. We assume the operator $\mathcal{O}_{k}$ has nonzero particle number. The case of operators carrying zero particle number will be discussed in section 5 .

Here, we recall the procedure for deriving these coefficients. The first few terms of the expansion (3.1) can be written as:

$$
\mathcal{O}_{2}(x) \mathcal{O}_{3}(0)=\left(C_{0}(x)+C_{1}^{i}(x) \partial_{i}+C_{2}(x) \partial_{t}+C_{3}^{i j}(x) \partial_{i} \partial_{j}+\cdots\right) \mathcal{O}_{1} .
$$

Commuting both sides with $K_{i}$, we get:

$$
\begin{aligned}
& \left(-i t \partial_{i}+N_{3} x_{i}\right) C_{0}=N_{1} C_{1}^{i}, \\
& \left(-i t \partial_{i}+N_{3} x_{i}\right) C_{1}^{j}=-i \delta_{i j} C_{2}+2 N_{1} C_{3}^{i j},
\end{aligned}
$$

while commuting with $C$ gives:

$$
\left(-i t^{2} \partial_{t}-i t x_{i} \partial_{i}-i t \Delta_{3}+\frac{x^{2}}{2} N_{3}\right) C_{0}=-i \Delta_{1} C_{2}+N_{1} C_{3}^{i i}
$$

These equations completley determine $C_{i}^{i}, C_{2}$, and $C_{3}^{i j}$ in terms of $C_{0}$ :

$$
\begin{aligned}
C_{1}^{i} & =\frac{1}{N_{1}}\left(-i t \partial_{i}+N_{3} x_{i}\right) C_{0} \\
C_{2} & =\frac{i}{N_{1}\left(2 \Delta_{1}-d\right)}\left[t^{2}\left(-2 i N_{1} \partial_{t}+\partial^{2}\right)-2 i N_{2} t x_{i} \partial_{i}+i\left(N_{3} d-2 N_{1} \Delta_{3}\right) t+N_{3} N_{2} x^{2}\right] C_{0} .
\end{aligned}
$$

Note that $N_{2}+N_{3}=N_{1}$. The expression for $C_{3}^{i j}$ in terms of $C_{0}$ can be easily written down using (3.3) and (3.5). The rest of the coefficients in the series can be derived in a similar fashion.

Operators with dimension $\boldsymbol{d} / \mathbf{2}$. From (3.5) it is clear that when the dimension of the operator $\mathcal{O}_{1}$ is equal to $d / 2$, the equation for $C_{2}$ becomes ill-defined and can be interpreted as a restriction on $C_{0}$ itself. We note that the value $d / 2$ is the unitarity bound on operator dimensions. In the theory of fermions at unitarity, the elementary fermion field $\psi$ and its Hermitian conjugate $\psi^{\dagger}$ have this dimension. In this case, we can identify $\mathcal{O}_{1}=\psi$, so $N_{1}=-1, \Delta_{1}=d / 2$, and $N_{3}=-\left(N_{2}+1\right)$. The equation for $C_{0}$ is then:

$$
\left[t^{2}\left(2 i \partial_{t}+\partial^{2}\right)-2 i N_{2} t x_{i} \partial_{i}+i\left(N_{3} d+2 \Delta_{3}\right) t+N_{3} N_{2} x^{2}\right] C_{0}=0 .
$$

This is a partial differential equation for $C_{0}$. However, scale invariance tells us that $C_{0}$ has the form:

$$
C_{0}(t, x)=t^{-\frac{1}{2} \Delta_{23,1}} f\left(x^{2} / t\right) .
$$

Using this form, the PDE for $C_{0}$ turns into an ordinary differential equation for $f$ :

$$
4 y f^{\prime \prime}+2\left[d-i\left(N_{2}-N_{3}\right) y\right] f^{\prime}+\left[N_{2} N_{3} y-i \frac{d}{2}\left(N_{2}-N_{3}\right)-i\left(\Delta_{2}-\Delta_{3}\right)\right] f=0 .
$$


The solution to this equation is

$$
f_{0}=e^{-\frac{i}{2} N_{3} y}\left[A U\left(-\alpha, \frac{d}{2},-\frac{i y}{2}\right)+B L_{\alpha}^{\frac{d}{2}-1}\left(-\frac{i y}{2}\right)\right]
$$

where $\alpha=\frac{1}{2}\left(\Delta_{2}-\Delta_{3}-\frac{d}{2}\right), U(a, b, x)$ is the confluent hypergeometric funtion, $L_{a}^{b}(x)$ is the generalized Laguerre polynomial and $A$ and $B$ are coefficients determined by the boundary conditions. Here, we note that regularity at $y \rightarrow 0$ sets $A$ equal to zero and $B$ can be fixed with proper normalization requirements.

The final result is that for the OPE of any two primary operators, the exact form of the coefficient of dimension $d / 2$ operators is known. In what follows, we discuss the consequences of this extra information.

It is worth noting that the fact that in equation (3.5), instead of deriving the coefficients of the descendants we ended up with a constraint on the coefficient of the primary is a reflection of the existence of a null operator (an operator which is both a primary and a descendant). Therefore, if we can find more null operators we would be able to impose more constraint equations for the OPE coefficients. This search can be done systematically by assuming the most general form of a descendant operator (an arbitrary number of raising operators $H$ and $P_{i}$ acting on a primary operator) and then requiring that the action of lowering operators $C$ and $K_{i}$ on this descendant operator gives zero. In this way, we can derive that all null operators with non-zero particle number are of the form $\left(\partial^{2}-2 i N \partial_{t}\right)^{n} \mathcal{O}$ where $\mathcal{O}$ is a primary of dimension $d / 2-n+1$ (This was first shown in [14]. For a recent review see [15]). In the case above $n=1$. Noting that $D=d / 2$ is the lowest scaling dimensions from unitarity constraints, we see that there is a unique null operator in any Schrödinger symmetric theory (more precisely, the number would be equal to the number of operators with scaling dimension $d / 2$ ). Again, the case of operators with zero particle number is unique and we examine it in section 5.

\section{Restrictions on multipoint correlators}

3-point Functions. One can use the known OPE coefficient to restrict the form of the 3 -point functions which include operators of dimension $d / 2$. From equation (2.7) we have:

$$
\lim _{x, t \rightarrow 0}\left\langle\mathcal{O}_{1}(y) \mathcal{O}_{2}(x) \mathcal{O}_{3}(0)\right\rangle=\left(t_{y}\right)^{-\Delta_{1}} t^{-\frac{1}{2} \Delta_{23,1}} \exp \left[\frac{i M_{1}}{2} \frac{y^{2}}{t_{y}}+\frac{i M_{2}}{2} \frac{x^{2}}{t}\right] F_{3}\left(x^{2} / t\right),
$$

where the fraction $x^{2} / t$ is kept finite in the limit. On the other hand from the OPE (3.2) we have:

$$
\lim _{x \rightarrow 0}\left\langle\mathcal{O}_{1}(y) \mathcal{O}_{2}(x) \mathcal{O}_{3}(0)\right\rangle=t^{-\Delta_{23,1} / 2} f\left(x^{2} / t\right)\left(t_{y}\right)^{-\Delta_{1}} \exp \left[i \frac{M_{1}}{2} \frac{y^{2}}{t_{y}}\right],
$$

where $f$ is defined in equation (3.7). From these we conclude that:

$$
F_{3}(z)=e^{-\frac{i}{2} M_{1} z} f(z)
$$


Therefore, if one of the operators has dimension $d / 2$, the form of the 3 -point function is completely known. We will identify $\mathcal{O}_{1}=\psi$, so $N_{1}=-1, \Delta_{1}=d / 2$, and $N_{3}=1-N_{2}$. There is one caveat here. The limits must all exist and be non-zero.

Now that we have the 3-point function exactly, it is easy to see that we can derive any of the OPEs involved by taking the appropriate limit. In particular, we can write:

$$
\psi(y) \mathcal{O}_{2}(x)=D_{0}(y-x) \mathcal{O}_{3}^{\dagger}(x) .
$$

Then, using the known result of the 3 point function discussed above, we can relate the coefficient $D_{0}$ to the coefficient $C_{0}$ in equation (3.2) by taking different limits. We have:

$$
C_{0}(x, t)=t^{\Delta_{1}-\Delta_{3}} \exp \left[i M_{2} \frac{x^{2}}{2 t}\right] D_{0}(x, t) .
$$

This can now be used to derive a differential equation for $D_{0}$ using (3.6):

$$
\left[2 i \partial_{t}+\partial^{2}\right] D_{0}(x, t)=0
$$

where we have used the scaling property of $D_{0}(x, t)$. We see that the OPE coefficient satisfies the Schrödinger equation. Moreover, we can also show that:

$$
\left[2 i M_{1} \partial_{t_{y}}+\partial_{y}^{2}\right]\left\langle\mathcal{O}_{1}(y) \mathcal{O}_{2}(x) \mathcal{O}_{3}(0)\right\rangle=0,
$$

whenever $\Delta_{1}=d / 2$ and $t_{y}>t_{x}>0$. Since the 3 -point function of primaries was already constrained to a function of a single variable, this extra constraint completely determines the function up to boundary conditions. In fact this explains why the 3-point functions of the elementary particle $\psi$, calculated in different theories $[5,9]$ match because they all satisfy this differential equation.

We could have expected this from the OPE expansion of the correlator. We note that the two point function of primary operators of scaling dimension $d / 2$ is the Green's function of the Schröedinger operator. Therefore, any n-point function that can be collapsed down to the two point function of these operators, will satisfy the Schröedinger equation. This is possible only when the operator with the scaling dimension $d / 2$ appears as either the first or last operator in a time-ordered correlator.

What happens when the operator appears in the middle of a correlator is not clear in general. However, in the special case of fermions at unitarity we can easily answer the question. The classical equation of motion states that:

$$
\left[\partial_{t}+\frac{1}{2 i M_{1}} \partial^{2}\right] \psi_{\uparrow}=\psi_{\downarrow}^{\dagger} \phi
$$

A simple Schwinger-Dyson type argument shows this result should hold inside correlators (upto contact terms). This is indeed true and in the case of 3-point functions can be checked explicitly using the differential equations derived above. We note that since the operator $\psi_{\downarrow}^{\dagger} \phi$ includes both annihilation and creation operators, its correlators would vanish if it appears at the endpoints, thus recovering the previous result.

We expect a similar relation to hold in general where the Schröedinger operator acting on a primary operator of scaling dimension $d / 2$ would equal a primary operator that includes both creation and annihilation operators. 
4-point Functions. Knowing the exact form of the 3-point functions, we can now try to restrict the form of the 4-point function. In particular, it seems that we are in a similar situation to relativistic CFTs, where the 3-point function is known and the 4-point can be viewed as a sum over intermediate states. The easiest way to calculate the form of the 4-point function when we have two operators of dimension $d / 2$ is to calculate it within a particular theory. Then by the previous results, the answer should be the same in any other theory with the same 3-point functions.

Here, we will use the embedding of $\mathfrak{s c h} \mathfrak{r}_{d}$ in $\mathfrak{c o n} \mathfrak{n}_{d+2}$ to carry out the calculation. This is a particularly nice choice, as the structure of 4-point function of the relativistic CFTs is known and the mapping between the 3-point functions is simple. Doing the calculation, we obtain:

$$
\begin{aligned}
\left\langle\mathcal{O}_{1} \mathcal{O}_{2} \mathcal{O}_{3} \mathcal{O}_{4}\right\rangle \sim & \delta\left(\sum M_{i}\right) \int \mathrm{d} \zeta_{1} \mathrm{~d} \zeta_{2} \mathrm{~d} \zeta_{3} e^{-i M_{1} \zeta_{1}-i M_{2} \zeta_{2}-i M_{3} \zeta_{3}} \times \\
& \times F\left(\frac{t_{12} t_{34}}{t_{13} t_{24}} \frac{\zeta_{3}\left(\zeta_{1}-\zeta_{2}+i v_{124}\right)}{\zeta_{2}\left(\zeta_{1}-\zeta_{3}+i v_{134}\right)}, \frac{t_{12} t_{34}}{t_{23} t_{14}} \frac{\zeta_{3}\left(\zeta_{1}-\zeta_{2}+i v_{124}\right)}{\zeta_{1}\left(\zeta_{2}-\zeta_{3}+i v_{234}\right)}\right) .
\end{aligned}
$$

We see that the general function $F_{4}$ of 4 variables is expressible as a function of only 2 variables. However, there is no simple relation between the two and one must use the integral formula above to relate them.

\section{Zero particle number sector and conservation laws}

In this section we look at the structure of the family associated with a primary $O$ with a zero particle number. What is special in this sector is the fact that the operators $K_{i}$ and $P_{j}$ that act as ladder operators, commute with each other. What this means is that descendants that we derive from raising the primary with the $P$ operator cannot be lowered back to the primary by the use of the lowering operator $K$ :

$$
K_{i} P_{j} O=P_{j} K_{i} O+\left[K_{i}, P_{j}\right] O=0 .
$$

Hence, if we have an operator $O_{i}^{\prime}$ which lowers to the primary operator $O$ with the use of $K_{i}$, this operator cannot be a descendant of $O$. We will call these operators which are not descendants of the primaries but nevertheless play a role in the operator algebra, alien operators.

We can derive a simple relation between the the family members (alien or descendant) at any level. Assuming $O^{(n)}$ is an alien operator at level n, which lowers to $O_{i}^{(n-1)}$, we have:

$$
P_{j} O_{i}^{(n-1)}=P_{j} K_{i} O^{(n)}=K_{i} P_{j} O^{(n)} .
$$

This is just the statement of the commutation of the ladder operators. Normally, the cartoon of a family tree would depict the ladder operators as parallel but in opposite directions. That is the operators $P K$ and $K P$, raising followed by lowering as well as lowering followed by raising, would act as identity. However, in the zero particle number case, $K_{i} P_{j}=P_{j} K_{i}$ and it is not proportional to the identity. If we orient the tree such that the scaling dimension changes vertically, $K_{i} P_{j}$ would act horizontally. 


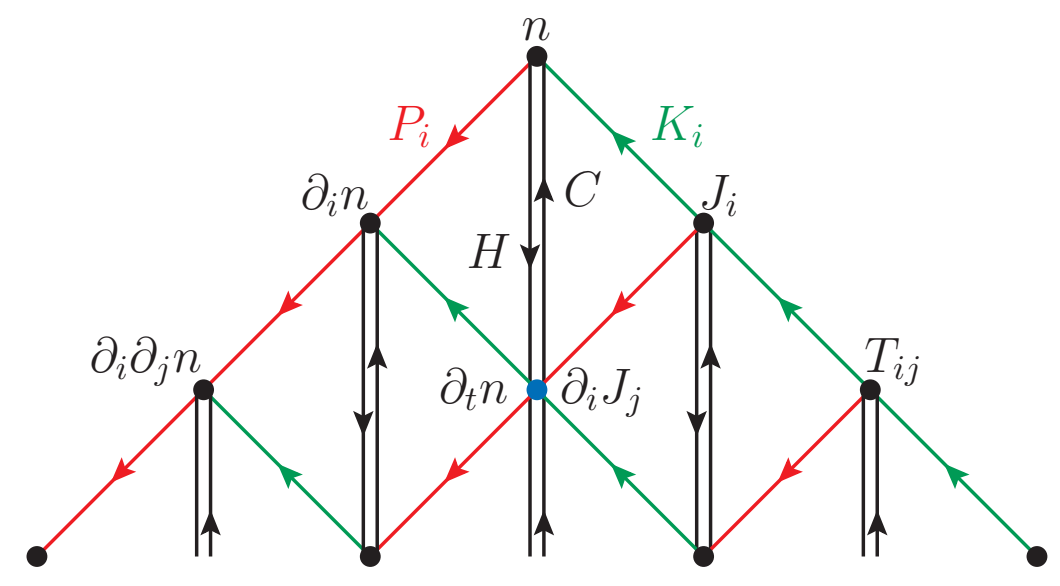

Figure 1. The family tree of the operator $n$.

As an example, we consider the particle number operator $n=\psi^{\dagger} \psi$, a primary with particle number zero. Using the notation $\overleftrightarrow{\partial_{i}}=\overrightarrow{\partial_{i}}-\overleftarrow{\partial_{i}}$, the first two alien operators are $J_{i}=-\frac{i}{2} \psi^{\dagger} \overleftrightarrow{\partial_{i}} \psi$ and $T_{i j}=\frac{-1}{4} \psi^{\dagger} \overleftrightarrow{\partial_{i}} \overleftrightarrow{\partial_{j}} \psi$, which appear on the far right at each level. ${ }^{1}$ Successive applications of $K_{i}$ and $P_{i}$ take us up or down along the tree but always slanted to the left (see figure 1). We note that by a simple counting argument, all possible spatial derivative combinations of $\psi$ and $\psi^{\dagger}$ appear on the tree.

In the family tree of a generic operator with particle number zero, each dot on the diagram represents only the position of an operator with respect to other operators. A remark is in order for when a spot on the diagram is occupied by more than one operator, which is the case for most positions. Two scenarios can arise. First, it is possible that there is no further relation between these operators than what is derived from their position on the family tree. That is, they are in fact distinct operators. This is the case for the descendants of a generic neutral operator.

However, it is also possible that there is a linear combination of the operators sitting on the same spot that lowers to zero using both $C$ and $K_{i}$. This is similar to the case of the null operator in the previous section. As an example, again we specialize to the family of the operator $n$ (However, the same arguments can be applied in any family where these requirements are met). Consider the spot denoted by the blue dot in figure 1. Here we have the two operators $\partial_{t} n$ and $\partial_{i} J_{j}$ which are of course related by the continuity equation $\partial_{t} n+\partial_{i} J_{i}=0$. And it is in fact this linear combination that is null. ${ }^{2}$

For the case of the primary operator $n$, it turns out that there is a single alien operator at each level of the family tree (e.g. $J_{i}$ and $T_{i j}$ for levels one and two) and that there exists a null operator (in these cases $\partial_{t} n+\partial_{i} J_{i}$ and $\partial_{t} J_{j}+\partial_{i} T_{i j}$ respectively.) Here, we give the

\footnotetext{
${ }^{1}$ We note that the operator $\psi^{\dagger} \overleftrightarrow{\partial_{t}} \psi$ is also an alien operator which lowers in the same way as $\delta^{i j} T_{i j}$ However, since there is a linear combination of the two which is a primary, we need only include one of the two in the family of the operator $n$ and of course we pick $T_{i j}$ since it carries more information than just its trace. A similar story happens at every level of the tree.

${ }^{2}$ However, because of the existence of the alien operator $T_{i j}$ this null combination does not restrict the form of the OPE as in the previous section.
} 
explicit form of the alien operator entering at level $l$ :

$$
C_{i_{1} \cdots i_{l}}^{(l)}=(2 i)^{-l} \psi^{\dagger} \overleftrightarrow{\partial_{i_{1}}} \cdots \overleftrightarrow{\partial_{i_{l}}} \psi
$$

We define $C^{(0)} \equiv n$ and note that $C_{i}^{(1)}=J_{i}$ and $C_{i j}^{(2)}=T_{i j}$. Using this definition, we have:

$$
\left[K_{k}, C_{i_{1} \cdots i_{l}}^{(l)}\right]=i \sum_{n=1}^{l} \delta_{k n} C_{i_{1} \cdots \bar{i}_{n} \cdots i_{l}}^{(l-1)}, \quad\left[C, C_{i_{1} \cdots i_{l}}^{(l)}\right]=0,
$$

where the notation $\overline{i_{n}}$ implies the $n$ 'th index is ommited. With these definitions for the alien operator at level $l$, we can easily derive that the following combination is null:

$$
O^{(l)}=\partial_{t} C_{i_{1} \cdots i_{l}}^{(l)}+\partial_{j} C_{j i_{1} \cdots i_{l}}^{(l+1)} .
$$

The fact that this null combination has the form of a conservation law is very suggestive. Whether or not the operator $O^{(l)}$ is in fact zero is another matter which cannot be answered by looking at the operator algebra alone. In general we can show that $O^{(1)}=O^{(2)}=0$, which are nothing but the continuity and energy conservation equations. In the free theory one can also demonstrate that the infinite conservation laws in this sector in fact arise from the $O^{(l)}$ 's [16]. It is also known that in the interacting theory all but the first few are broken.

What the algebra does demonstrate is that if there are conservation laws, they should be found among the null operators which relate the alien operators of adjacent levels. And in fact if there is a conservation law in the free theory that is broken in the interacting theory, its non-conservation must act as a primary operator. That is, its n-point functions with other primaries are restricted as in equations (2.6) and (2.7).

\section{Conclusion}

We have shown that it is possible to further restrict the OPE and n-point functions of some primary operators in CFTs just by algebra considerations. In particular, we showed that operators with critical scaling dimension $d / 2$, e.g. the elementary particle $\psi$, has known OPE with any other primary. Because of this, its 3-point function is determined up to an overall constant.

We also analyzed the structure of the descendants of primaries with zero particle number and showed that there are non-descendant operators that nevertheless play an important role in the family, the so-called alien operators. There is an intimate relationship between null operators derived from these alien operators and conservation laws. However, the question of conservation is one that needs to be looked at in each theory.

\section{Acknowledgments}

This work is supported, in part, by DOE grant DE-FG02-13ER41958. The authors thank Sergej Moroz for extensive discussion and comments. 
Open Access. This article is distributed under the terms of the Creative Commons Attribution License (CC-BY 4.0), which permits any use, distribution and reproduction in any medium, provided the original author(s) and source are credited.

\section{References}

[1] C.R. Hagen, Scale and conformal transformations in galilean-covariant field theory, Phys. Rev. D 5 (1972) 377 [INSPIRE].

[2] U. Niederer, The maximal kinematical invariance group of the free Schrödinger equation., Helv. Phys. Acta 45 (1972) 802 [inSPIRE].

[3] T. Mehen, I.W. Stewart and M.B. Wise, Conformal invariance for nonrelativistic field theory, Phys. Lett. B 474 (2000) 145 [hep-th/9910025] [INSPIRE].

[4] M. Henkel, Schrödinger invariance in strongly anisotropic critical systems, J. Statist. Phys. 75 (1994) 1023 [hep-th/9310081] [INSPIRE].

[5] M. Henkel and J. Unterberger, Schrödinger invariance and space-time symmetries, Nucl. Phys. B 660 (2003) 407 [hep-th/0302187] [INSPIRE].

[6] Y. Nishida and D.T. Son, Nonrelativistic conformal field theories, Phys. Rev. D 76 (2007) 086004 [arXiv: 0706 . 3746] [INSPIRE].

[7] D.T. Son, Toward an AdS/cold atoms correspondence: A Geometric realization of the Schrödinger symmetry, Phys. Rev. D 78 (2008) 046003 [arXiv: 0804.3972] [INSPIRE].

[8] K. Balasubramanian and J. McGreevy, Gravity duals for non-relativistic CFTs, Phys. Rev. Lett. 101 (2008) 061601 [arXiv: 0804.4053] [INSPIRE].

[9] C.A. Fuertes and S. Moroz, Correlation functions in the non-relativistic AdS/CFT correspondence, Phys. Rev. D 79 (2009) 106004 [arXiv:0903.1844] [INSPIRE].

[10] A. Volovich and C. Wen, Correlation Functions in Non-Relativistic Holography, JHEP 05 (2009) 087 [arXiv: 0903.2455] [INSPIRE].

[11] W. Zimmermann, Local operator products and renormalization in quantum field theory, in Lectures on Elementary Particles and Quantum Field Theory, The MIT Press (1971) [ISBN-13: 978-0262540131].

[12] K.G. Wilson, Renormalization group and critical phenomena. 2. Phase space cell analysis of critical behavior, Phys. Rev. B 4 (1971) 3184 [INSPIRE].

[13] K.G. Wilson and W. Zimmermann, Operator product expansions and composite field operators in the general framework of quantum field theory, Commun. Math. Phys. 24 (1972) 87 [INSPIRE] and online at http://projecteuclid.org/euclid.cmp/1103857739.

[14] V.K. Dobrev, H.-D. Doebner and C. Mrugalla, Lowest weight representations of the Schrödinger algebra and generalized heat/Schrödinger equations, Rep. Math. Phys. 39 (1997) 201.

[15] V.K. Dobrev, Non-Relativistic Holography - A Group-Theoretical Perspective, Int. J. Mod. Phys. A 29 (2014) 1430001 [arXiv:1312.0219] [InSPIRE].

[16] X. Bekaert, E. Meunier and S. Moroz, Symmetries and currents of the ideal and unitary Fermi gases, JHEP 02 (2012) 113 [arXiv:1111.3656] [INSPIRE]. 